\title{
Plasma p tau 181 levels is associated with white matter microstructural changes in across the Alzheimer's disease stages
}

Fardin Nabizadeh ( $\square$ fardinnabizade1378@gmail.com )

Neuroscience Research Group (NRG), Universal Scientific Education and Research Network (USERN), Tehran, Iran

Seyed Behnamedin Jameie

Neuroscience Research Center (NRC), Iran University of Medical Science, Tehran, Iran

\section{Saghar Khani}

Iran university of Medical science

\section{Aida Rezaei}

School of Medicine, Tehran University of Medical Science, Tehran, Iran

\section{Fatemeh Ranjbaran}

Iran university of Medical science

\section{Niloofar Deravi}

Student Research committee, School of medicine, Shahid Beheshti University of Medical Science,

Tehran, Iran

\section{Research Article}

Keywords: Alzheimer's Disease (AD), p tau 181, microstructural change

Posted Date: December 5th, 2020

DOI: https://doi.org/10.21203/rs.3.rs-115316/v2

License: (c) (1) This work is licensed under a Creative Commons Attribution 4.0 International License. Read Full License 


\section{Abstract}

Alzheimer's Disease (AD) is characterized by cognitive impairments and memory difficulties, which hinder daily activities and lead to personal and behavioral problems. In recent years, blood-based biomarkers like plasma phosphorylated tau protein at threonine 181 ( $p$ tau 181) emerged as new tools and showed sufficient power in detecting AD patients from healthy people. Here we investigate the correlation between $\mathrm{p}$ tau 181 and white matter microstructural changes in AD. We add 41 patients diagnosed with Alzheimer's, 119 patients with mild cognitive impairments and 43 healthy controls with baseline plasma $p$ tau 181 level and DTI values for each region of interest from the ADNI database. The analysis revealed that the plasma level of $p$ tau 181 could predict changes of MD (Mean Diffusivity), RD (Radial Diffusivity), DA (Axial Diffusivity) and FA (Fractional Anisotropy) parameters in widespread regions and there is a significant association between white matter pathway alteration in different regions and $p$ tau 181 plasma measurements within each group. In conclusion, our findings showed that plasma $p$ tau 181 levels are associated with cellular and molecular changes in AD, which enhance the biomarkers for diagnostic procedures and support the application of plasma $\mathrm{p}$ tau 181 as a biomarker for white matter changes and neurodegeneration. Longitudinal studies are also necessary for proving the efficacy of these biomarkers and predicting the role in structural changes.

\section{Introduction}

Alzheimer's disease (AD) is responsible for the course of dementia and memory deficit, and eventually leads to cognitive and functional decline, affecting millions of elderly people across the globe $(1,2)$. AD is characterized by cognitive impairments and memory difficulties, which hinder daily activities, and cause personal and behavioural problems $(1,3)$. $A D$ is associated with the formation of neurofibrillary tangles (NFTs), including hyperphosphorylated tau protein ( $p$ tau) and extracellular Amyloid $\beta(A \beta)$ plaques in regions responsible for memory and other cognitive functions such as the hippocampus $(4,5)$. NFTs are typical brain lesions consisting of aggregated and hyperphosphorylated forms of tau protein, which lead to loss of the ability to bind microtubules and are assembled into paired helical filaments. Intracellular NFTs in regions involved with cognitive functions are associated with cognitive decline by the disruption in axonal transport and neural loss $(6,7)$.

Many researchers reported $A \beta$, total Tau and Tau phosphorylated at threonine 181 ( $p$ tau 181) in cerebrospinal fluid (CSF), and Positron emission tomography (PET) as biomarkers for Alzheimer's diagnoses. However, in recent years plasma biomarkers emerged as new diagnostic tools and showed sufficient efficacy in detecting AD patients from healthy people (8). Blood-based measures showed that $p$ tau 181 might be a reliable biomarker for Alzheimer's and disease progression (9-11). However, there is no evidence that shows the effect of plasma $p$ tau 181 on white matter connections and neurodegeneration in the brain of AD patients. We hypothesized plasma $p$ tau 181 level could predict changes in structural brain connections in regions that play a significant role in cognitive, learning and memory function. In the present research, we investigated the correlation between $p$ tau 181 in serum and diffusion tensor imaging (DTI) values in an observational cross-sectional study to address this question. 


\section{Materials And Methods}

\section{Data Acquisition}

Participants' information was acquired from the Alzheimer's Disease Neuroimaging Initiative (ADNI) database (adni.loni.usc.edu). The ADNI led by Principal Investigator Michael W. Weiner, MD was launched in 2003 as a public-private partnership. The primary goal of ADNI has been to test whether serial magnetic resonance imaging (MRI), positron emission tomography (PET), other biological markers, and clinical and neuropsychological assessment can be combined to measure the progression of mild cognitive impairment (MCl) and early Alzheimer's disease (AD). We extracted baseline visit data for which demographic data, post-processed DTI, and plasma $p$ tau 181 tau levels from baseline visit were available. We also downloaded results of white matter hyperintensity analysis and brief volumetric data for better understanding. Our cross-sectional study consisted of 21 patients with $A D$, with their baseline plasma $p$ tau 181, CSF Amyloid $\beta$, CSF total tau, and CSF $p$ tau levels and their post-processed Diffusion Tensor Imaging (DTI).

Participants were classified as AD patients if their mini-mental state examination (MMSE) was between 20 and 24, clinical dementia rating (CDR) score was between 0.5 and 1, and met the National Institute of Neurologic and Communicative Disorders and Stroke and the Alzheimer's Disease and Related Disorders Association criteria (12).

\section{Plasma p tau 181 Measurements}

Plasma samples were analyzed at the University of Gothenburg, Sweden by using the Single-Molecule array (Simoa) technique. The detailed procedure is described in (adni.loni.usc.edu). (11).

\section{DTI Imaging Processing}

We extracted the results of DTI ROI analysis from ADNI. For each participant, all images were corrected, normalized and extracerebral tissue was removed by the Extraction Tool (BET) in FSL (13). To align data from different subjects into the same 3D coordinate space, each T1- weighted anatomical image was linearly aligned to a version of the Colins27 brain template (14) using FSL's flirt (15) with 6 degrees of freedom to allow translations and rotations in 3D. The Colin27 brain was zero-padded to have a cubic isotropic image size $\left.220 \times 220 \times 2201 \mathrm{~mm}^{\wedge} 3\right)$ and then downsampled $\left(110 \times 110 \times 1102 \mathrm{~mm}^{\wedge} 3\right)$ to be more similar to the DWI resolution.

To adjust echo-planar imaging (EPI) induced susceptibility artifacts, which can cause distortions at tissue-fluid interfaces, skull-stripped b0 images were linearly aligned to their respective T1-weighted structural scans using FSL's flirt with 9 degrees of freedom and then elastically registered to their aligned T1 scans using an inverse consistent registration algorithm with a mutual information cost function (16) as described in (17). The resulting 3D deformation fields were then applied to the remaining $41 \mathrm{DWI}$ volumes before mapping diffusion parameters. To account for linearly registering the average b0 from the DWI images to the structural T1-weighted scan, a corrected gradient table was calculated. 
A single diffusion tensor was modeled at each voxel in the brain from the eddy- and EPI-corrected DWI scans using FSL's dtifit command, and scalar anisotropy and diffusivity maps were obtained from the resulting diffusion tensor eigenvalues $(\lambda 1, \lambda 2, \lambda 3)$. Fractional anisotropy (FA) was calculated from the standard formula.

We registered the FA image from the JHU DTI atlas (18) to each subject using a previously described mutual information-based elastic registration algorithm(16). We then applied the deformation to the stereotaxic JHU “Eve” WM atlas labels (http://cmrm.med.jhmi.edu/cmrm/atlas/human_data/file/Atlas Explanation2.htm) using nearest neighbour interpolation to avoid intermixing of the labels.

This placed the atlas ROIs in the same coordinate space as our DTI maps. We were then able to calculate the average FA and MD within the boundaries of each of the ROI masks for each subject. Of the $56 \mathrm{WM}$ ROIs, we excluded 4 ROIs, the left and right middle cerebellar peduncle, and the pontine crossing tract, as they often fall wholly or partially out of the field of view (FOV). We note that this is also occasionally true of the left and right medial lemniscus, inferior and superior peduncles. We only included non-zero voxels within the FOV in our calculations of mean FA and MD. In addition to the $52 \mathrm{JHU}$ labels, five more ROIs were evaluated: the bilateral fornix, bilateral genu, bilateral body, and bilateral splenium of the corpus callosum and the full corpus callosum, to get full summary measures of the regions.

Tensor based spatial statistics (19) was also performed, and the mean FA in regions of interest along the skeleton was extracted. TBSS was performed according to protocols outlined by the ENIGMA-DTI group: http://enigma.loni.ucla.edu/wpcontent/uploads/2012/06/ENIGMA_TBSS_protocol.pdf

In short, all subjects were registered to the ENIGMA-DTI template in ICBM space, and standard tbss steps were performed to project individual FA maps onto the skeletonized ENIGMA-DTI template. ROI extraction was also performed according to the following protocol to extract the mean FA in ROls along with the skeleton: http://enigma.loni.ucla.edu/wpcontent/uploads/2012/06/ENIGMA_ROI_protocol.pdf.

\section{Cognitive assessments}

The patients' cognitive condition was assessed by the Mini-Mental State Exam (MMSE), which is a common test of cognitive function among the elderly, including tests of orientation, attention, memory, language, and visual-spatial skills. MMSE scores were extracted for each patient from the ADNI MiniMental Examination.

\section{Statistical Analyses}

We used SPSS16 software for statistical analyses. First we tested the normality of variables by Kolmogorov Smirnov and Shapiro-Wilk tests. Then we log-transformed the non-normal variables to normal distribution. We analyzed the difference between groups by one-way ANOVA using Bonferroni correction for multiple comparisons. Then we used a partial correlation adjusted for age, sex, and APOE for checking the relation between plasma $p$ tau 181 and other demographic variables once among all participants and then within groups. We used the same model for investigating the association between 
plasma $\mathrm{p}$ tau 181 and DTI values in each ROI. The bootstrap method was used for addressing type I error due to multiple comparisons in the correlation models.

\section{Result}

\section{Patient characteristics}

In this study, baseline cohort information of 203 participants was entered. The mean age was 73, including 117 men and 86 women. Participants were all educated and the average length of their education was 15.9 years. Among all participants, 102 had at least one APOE $\varepsilon 4$. The mean scores of the MMSE test were 27.25, and details of demographic information for each group are provided in Table 1.

\section{Between groups analysis}

The level of plasma $p$ tau 181 is significantly different between the groups $[F(2,200)=9.05, p=0.000]$ and there is no meaningful difference between the group's age and mean education time $[F(2,200)=0.425$, $p=0.655],[F(2,200)=2.50, p=0.084]$. The comparison of APOE genotype status between groups revealed a strong difference $[F(2,200)=9.06, p=0.000]$, and the glucose uptake was significantly lower in the $A D$ group $[F(2,200)=43.9, p=0.000]$.

\section{Plasma p tau 181 with characteristics}

The partial correlation model for the $p$ tau 181 level and each demographic variable, while adjusted for age, sex, and APOE genotype showed that plasma $p$ tau 181 has a strong correlation with glucose uptake in angular, temporal, and posterior cingulate [correlation coefficient:-0.231, p: 0.001], education time [correlation coefficient:-0.166, p: 0.019], and MMSE score [correlation coefficient:-0.216, p: 0.002]. In addition, there is a positive correlation between age and $p$ tau while controlled for sex and APOE [correlation coefficient: 0.215 , p: 0.002] among all participants. When we investigated the correlation between $p$ tau 181 and demographic features within groups, the correlations started to disappear, and there is only one correlation between $p$ tau and glucose uptake in AD patients [correlation coefficient:-0.397, p: 0.017].

\section{Plasma p tau 181 with microstructural changes}

Initially, we assessed the relation between $\mathrm{p}$ tau level and DTI values in each ROI among all participants by implementing the partial correlation model adjusted for effects of age, sex, and APOE genotype. There is a strong correlation between plasma measures of $p$ tau 181 with a widespread pattern of changes in each FA, DA, RD, and MD in the brain (Tab2). We found a negative correlation between $p$ tau and FA in the left hippocampal cingulum, Splenium of the right corpus callosum, Left and Right Tapatum. Moreover, a higher level of $p$ tau 181 correlates with the overall higher MD and DA in the Splenium of the right and left corpus callosum, Left and Right Tapatum, Right Posterior corona radiate, Right Sagittal stratum, Right Uncinate fasciculus, Retrolenticular part of the right internal capsule, and Left Medial lemniscus (Tab2). Moreover, the correlation was the same for RD in the left hippocampal cingulum, Splenium of the right 
corpus callosum, Left and Right Tapatum, Right Posterior corona radiate, Right Sagittal stratum, Right Uncinate fasciculus, Retrolenticular part of the right internal capsule, and Left Medial lemniscus (Table 2).

The investigated relation between $\mathrm{p}$ tau 181 and connectometry values within each group showed there is a significant correlation for MD, RD, and DA in the Left Medial lemniscus, MD and DA in the Left Inferior cerebellar peduncle, MD and RD in the Right Superior cerebellar peduncle among AD patients (Table 3 ). The $\mathrm{p}$ tau correlates with MD and RD, only in the Left Medial lemniscus within the MCl group (Table 3). Partial correlation revealed that there is a correlation between $p$ tau 181 and DA in the Right Superior cerebellar peduncle and RD in the Left Uncinate fasciculus among healthy controls (Table 3).

\section{Discussion}

In a cross-sectional study based on the ADNI cohort, it was concluded that the plasma level of $p$ tau 181 independently predicts microstructural changes in the brain of Alzheimer's patients. Using a step-by-step strategy, we initially investigated the difference between participant's demographic information, including age, glucose uptake, MMSE scores, education, APOE genotype, sex, and plasma $p$ tau 181. Then we narrowed the study to examine the correlation between $p$ tau 181 with demographic variables among all participants and within groups separately. Afterwards, we used a partial correlation model controlled for age, APOE and sex to investigate the relation between $p$ tau 181 in plasma and changes in white matter. Baseline plasma $p$ tau 181 levels are associated with widespread white matter changes in all participants in the disease's pathological signature areas, including the hippocampal cingulum, Splenium of corpus callosum, Tapatum, Posterior corona radiate, Sagittal stratum, Uncinate fasciculus, Retrolenticular part of the internal capsule, cerebellar peduncles, and Medial lemniscus. Overall, according to observations, a decrease in FA and increase in MD reflects demyelination and axonal loss (20). This is the first study to investigate the relationship between plasma $p$ tau 181 and white matter changes.

In the onset of dementia and cognitive decline, many areas seem to change. The internal capsule, corona radiates, Uncinate fasciculus, cerebellar peduncles, Medial lemniscus, and hippocampal cingulum in AD patients change compared to healthy people without cognitive problems (21). In our study, plasma p tau 181 levels were significantly associated with these areas, indicating that our findings are in line with previous studies investigating microstructural changes related to Alzheimer's. As a result of our analysis, plasma $\mathrm{p}$ tau 181 predicted structural changes in the left hippocampal cingulum, which indicates the importance of the cingulum as an important area in the pathological course of the disease, and there is also evidence of a link between CSF $p$ tau and $A \beta$ with a change of MD in the cingulum region (22). Moreover, the research results provide compelling evidence in support of our findings and state that brain connectivity in the posterior cingulum can be a good predictor for cognitive decline in Alzheimer's disease (23). The cingulum bundle is an important white matter tract that connects the frontal, parietal, and medial temporal, linking the subcortical nucleus to the cingulate gyrus, extending into the hippocampal and parahippocampal regions. Consequently, damage in areas close to the hippocampus in the cingulum causes cognitive problems in many domains such as language, memory and executive control (24). 
Previously, CSF biomarkers were revealed as a predictor for changes in the Uncinate fasciculus (25) and these regions involved in language processing and damage to uncinate fasciculus may cause language impairments (26). Meanwhile, there is a significant correlation between plasma $p$ tau 181 and changes in this region from our results. Similarly, X Li et al. found that pathological levels of AB42 and CSF total tau in people with Alzheimer's-related cognitive impairments correlated with decreased FA and increased MD in the white matter pathway (27).

Biomarkers in CSF may be active several years before the onset of symptoms, while $A \beta$ and Tau are the most critical (28). Many studies have emphasized the diagnostic role of $t$ tau and $p$ tau in CSF and state that they can predict the progression to dementia (29-32). On the other hand, some studies present different findings $(33,34)$. Despite this, in predicting AD by each of these biomarkers alone, $p$-tau is preferred in terms of specificity and sensitivity (28).

Studies have been conducted on blood-based biomarkers in recent years as a noninvasive and accessible marker for monitoring people with a risk of developing Alzheimer's. Evidence revealed that plasma $\mathrm{p}$ tau 181 and $t$ tau levels have a high diagnostic value, and their levels are much higher in AD subjects than in $\mathrm{MCl}$ and healthy control (35). However, studies have described $\mathrm{p}$ tau 181 to be better than $\mathrm{t}$ tau $(9,10)$. The largest plasma $p$ tau 181 study in the diagnosis of Alzheimer's, which included the results of four independent cohorts, states that plasma $p$ tau 181 has a high performance in identifying the clinical diagnosis of Alzheimer's patients with an unknown amyloid status and was able to differentiate Alzheimer's disease from other neurodegenerative diseases and can detect Alzheimer's in the early stages using $A \beta$ PET (11).

In conclusion, our study results show that plasma $\mathrm{p}$ tau 181 levels are associated with neurodegeneration in pathogenesis regions of Alzheimer's disease, which enhance this biomarker's diagnostic status and support the application of blood-based biomarkers as an early detector for white matter damages. Due to the increasing population being diagnosed with Alzheimer's and the resulting social costs and considering that $A D$ 's pathogenesis exists several years before its clinical signs, achieving a reliable biomarker with adequate sensitivity and specificity is necessary. Although plasma $p$ tau 181 is superior to CSF biomarkers and imaging techniques in terms of availability, low cost, and non-invasiveness, further efforts should be made to standardize the biomarkers' measurement and define their pathological threshold. Longitudinal studies are also necessary to prove the efficacy of this biomarker's predicting role in structural changes.

\section{Declarations}

* Data used in the preparation of this article were obtained from the Alzheimer's disease Neuroimaging Initiative (ADNI) database (adni.loni.usc.edu). As such, the investigators within the ADNI contributed to the design and implementation of $\mathrm{ADNI}$ and/or provided data but did not participate in the analysis or writing of this report. A complete listing of ADNI investigators can be found at:

http://adni.loni.usc.edu/wp-content/uploads/how_to_apply/ADNI_Acknowledgement_List.pdf 
We have no conflicts of interest to disclose.

\section{Acknowledgements}

Data collection and sharing for this project was funded by the Alzheimer's Disease Neuroimaging Initiative (ADNI) (National Institutes of Health Grant U01 AG024904) and DOD ADNI (Department of Defense award number W81XWH-12-2-0012). ADNI is funded by the National Institute on Aging, the National Institute of Biomedical Imaging and Bioengineering, and through generous contributions from the following: AbbVie, Alzheimer's Association; Alzheimer's Drug Discovery Foundation; Araclon Biotech; BioClinica, Inc.; Biogen; Bristol-Myers Squibb Company; CereSpir, Inc.; Cogstate; Eisai Inc.; Elan Pharmaceuticals, Inc.; Eli Lilly and Company; Eurolmmun; F. Hoffmann-La Roche Ltd and its affiliated company Genentech, Inc.; Fujirebio; GE Healthcare; IXICO Ltd.; Janssen Alzheimer Immunotherapy Research \& Development, LLC.; Johnson \& Johnson Pharmaceutical Research \& Development LLC.; Lumosity; Lundbeck; Merck \& Co., Inc.; Meso Scale Diagnostics, LLC.; NeuroRx Research; Neurotrack Technologies; Novartis Pharmaceuticals Corporation; Pfizer Inc.; Piramal Imaging; Servier; Takeda Pharmaceutical Company; and Transition Therapeutics. The Canadian Institutes of Health Research is providing funds to support ADNI clinical sites in Canada. Private sector contributions are facilitated by the Foundation for the National Institutes of Health (www.fnih.org). The grantee organization is the Northern California Institute for Research and Education, and the study is coordinated by the Alzheimer's Therapeutic Research Institute at the University of Southern California. ADNI data are disseminated by the Laboratory for Neuro Imaging at the University of Southern California.

\section{References}

1. Mantzavinos V, Alexiou A. Biomarkers for Alzheimer's Disease Diagnosis. Curr Alzheimer Res. 2017;14(11):1149-54.

2. Oboudiyat C, Glazer H, Seifan A, Greer C, Isaacson RS. Alzheimer's disease. Semin Neurol. 2013;33(4):313-29.

3. Weller J, Budson A. Current understanding of Alzheimer's disease diagnosis and treatment. F1000Res. 2018;7:F1000 Faculty Rev-161.

4. Wu XL, Piña-Crespo J, Zhang YW, Chen XC, Xu HX. Tau-mediated Neurodegeneration and Potential Implications in Diagnosis and Treatment of Alzheimer's Disease. Chin Med J (Engl). 2017;130(24):2978-90.

5. Kandimalla R, Manczak M, Yin X, Wang R, Reddy PH. Hippocampal phosphorylated tau induced cognitive decline, dendritic spine loss and mitochondrial abnormalities in a mouse model of Alzheimer's disease. Hum Mol Genet. 2018;27(1):30-40.

6. Rissman RA. Stress-induced tau phosphorylation: functional neuroplasticity or neuronal vulnerability? J Alzheimers Dis. 2009;18(2):453-7.

7. Hampel H, Blennow K, Shaw LM, Hoessler YC, Zetterberg H, Trojanowski JQ. Total and phosphorylated tau protein as biological markers of Alzheimer's disease. Exp Gerontol. 
2010;45(1):30-40.

8. Olsson B, Lautner R, Andreasson U, Öhrfelt A, Portelius E, Bjerke M, et al. CSF and blood biomarkers for the diagnosis of Alzheimer's disease: a systematic review and meta-analysis. Lancet Neurol. 2016;15(7):673-84.

9. Mielke MM, Hagen CE, Xu J, Chai X, Vemuri P, Lowe VJ, et al. Plasma phospho-tau181 increases with Alzheimer's disease clinical severity and is associated with tau- and amyloid-positron emission tomography. Alzheimers Dement. 2018;14(8):989-97.

10. Yang CC, Chiu MJ, Chen TF, Chang HL, Liu BH, Yang SY. Assay of Plasma Phosphorylated Tau Protein (Threonine 181) and Total Tau Protein in Early-Stage Alzheimer's Disease. Journal of Alzheimer's disease : JAD. 2018;61(4):1323-32.

11. Karikari TK, Pascoal TA, Ashton NJ, Janelidze S, Benedet AL, Rodriguez JL, et al. Blood phosphorylated tau 181 as a biomarker for Alzheimer's disease: a diagnostic performance and prediction modelling study using data from four prospective cohorts. The Lancet Neurology. 2020;19(5):422-33.

12. McKhann G, Drachman D, Folstein M, Katzman R, Price D, Stadlan EM. Clinical diagnosis of Alzheimer's disease: report of the NINCDS-ADRDA Work Group under the auspices of Department of Health and Human Services Task Force on Alzheimer's Disease. Neurology. 1984;34(7):939-44.

13. Smith SM. Fast robust automated brain extraction. Hum Brain Mapp. 2002;17(3):143-55.

14. Holmes CJ, Hoge R, Collins L, Woods R, Toga AW, Evans AC. Enhancement of MR images using registration for signal averaging. J Comput Assist Tomogr. 1998;22(2):324-33.

15. Jenkinson M, Bannister P, Brady M, Smith S. Improved optimization for the robust and accurate linear registration and motion correction of brain images. Neuroimage. 2002;17(2):825-41.

16. Leow AD, Yanovsky I, Chiang MC, Lee AD, Klunder AD, Lu A, et al. Statistical properties of Jacobian maps and the realization of unbiased large-deformation nonlinear image registration. IEEE Trans Med Imaging. 2007;26(6):822-32.

17. Jahanshad N, Lee AD, Barysheva M, McMahon KL, de Zubicaray GI, Martin NG, et al. Genetic influences on brain asymmetry: a DTI study of 374 twins and siblings. Neuroimage. 2010;52(2):45569.

18. Mori S, Oishi K, Jiang H, Jiang L, Li X, Akhter K, et al. Stereotaxic white matter atlas based on diffusion tensor imaging in an ICBM template. Neuroimage. 2008;40(2):570-82.

19. Smith SM, Jenkinson M, Johansen-Berg H, Rueckert D, Nichols TE, Mackay CE, et al. Tract-based spatial statistics: voxelwise analysis of multi-subject diffusion data. Neuroimage. 2006;31(4):1487505.

20. Kantarci K. Fractional anisotropy of the fornix and hippocampal atrophy in Alzheimer's disease. Frontiers in aging neuroscience. 2014;6:316.

21. Mayo CD, Mazerolle EL, Ritchie L, Fisk JD, Gawryluk JR. Longitudinal changes in microstructural white matter metrics in Alzheimer's disease. Neurolmage Clinical. 2017;13:330-8. 
22. Racine AM, Merluzzi AP, Adluru N, Norton D, Koscik RL, Clark LR, et al. Association of longitudinal white matter degeneration and cerebrospinal fluid biomarkers of neurodegeneration, inflammation and Alzheimer's disease in late-middle-aged adults. Brain Imaging Behav. 2019;13(1):41-52.

23. Nakata Y, Sato N, Nemoto K, Abe O, Shikakura S, Arima K, et al. Diffusion abnormality in the posterior cingulum and hippocampal volume: correlation with disease progression in Alzheimer's disease. Magnetic resonance imaging. 2009;27(3):347-54.

24. Bubb EJ, Metzler-Baddeley C, Aggleton JP. The cingulum bundle: Anatomy, function, and dysfunction. Neuroscience \& Biobehavioral Reviews. 2018;92:104-27.

25. Drummond C, Coutinho G, Monteiro MC, Assuncao N, Teldeschi A, de Souza AS, et al. Narrative impairment, white matter damage and CSF biomarkers in the Alzheimer's disease spectrum. Aging. 2019;11(20):9188-208.

26. Friederici $A D$. The brain basis of language processing: from structure to function. Physiological reviews. 2011;91(4):1357-92.

27. Li X, Li TQ, Andreasen N, Wiberg MK, Westman E, Wahlund LO. The association between biomarkers in cerebrospinal fluid and structural changes in the brain in patients with Alzheimer's disease. Journal of internal medicine. 2014;275(4):418-27.

28. Tan CC, Yu JT, Tan L. Biomarkers for preclinical Alzheimer's disease. Journal of Alzheimer's disease : JAD. 2014;42(4):1051-69.

29. Lewczuk P, Lelental N, Lachmann I, Holzer M, Flach K, Brandner S, et al. Non-Phosphorylated Tau as a Potential Biomarker of Alzheimer's Disease: Analytical and Diagnostic Characterization. Journal of Alzheimer's disease : JAD. 2017;55(1):159-70.

30. Blennow K, Vanmechelen E, Hampel H. CSF total tau, Abeta42 and phosphorylated tau protein as biomarkers for Alzheimer's disease. Molecular neurobiology. 2001;24(1-3):87-97.

31. Schönknecht P, Pantel J, Hunt A, Volkmann M, Buerger $K$, Hampel $H$, et al. Levels of total tau and tau protein phosphorylated at threonine 181 in patients with incipient and manifest Alzheimer's disease. Neuroscience letters. 2003;339(2):172-4.

32. Lewczuk P, Esselmann H, Bibl M, Beck G, Maler JM, Otto M, et al. Tau protein phosphorylated at threonine 181 in CSF as a neurochemical biomarker in Alzheimer's disease: original data and review of the literature. Journal of molecular neuroscience : MN. 2004;23(1-2):115-22.

33. Rizzi L, Missiaggia L, Roriz-Cruz M. CSF Aß(1-42), but not p-Tau(181), Predicted Progression from Amnestic MCl to Alzheimer's Disease Dementia. Neuromolecular medicine. 2018;20(4):491-7.

34. Haense C, Buerger K, Kalbe E, Drzezga A, Teipel SJ, Markiewicz P, et al. CSF total and phosphorylated tau protein, regional glucose metabolism and dementia severity in Alzheimer's disease. European journal of neurology. 2008;15(11):1155-62.

35. Shekhar S, Kumar R, Rai N, Kumar V, Singh K, Upadhyay AD, et al. Estimation of Tau and Phosphorylated Tau181 in Serum of Alzheimer's Disease and Mild Cognitive Impairment Patients. PLoS One. 2016;11(7):e0159099-e. 


\section{Tables}

TABLE1. Participant's characteristics

\begin{tabular}{lllll}
\hline Demographic and health characteristics & $\mathrm{CN}(\mathbf{4 3})$ & $\mathrm{MCI}(119)$ & $\mathrm{AD}(41)$ & P value \\
\hline Age, years & $72.9( \pm 6.2)$ & $72.8( \pm 6.8)$ & $74.0( \pm 8.6)$ & 0.655 \\
Sex(M/F) & $22 / 21$ & $71 / 48$ & $24 / 17$ & 0.625 \\
Education, years & $16.4( \pm 2.6)$ & $16.0(2.6)$ & $15.2( \pm 2.9)$ & 0.084 \\
MMSE & $28.8( \pm 1.4)$ & $27.9( \pm 1.9)$ & $23.4( \pm 1.8)$ & 0.000 \\
FDG-PET & $1.30( \pm 0.14)$ & $1.29( \pm 0.12)$ & $1.06( \pm 0.16)$ & 0.000 \\
APOE genotype & & & & 0.000 \\
With out $\varepsilon 4$ & 31 & 58 & 12 & \\
One $\varepsilon 4$ & 12 & 49 & 23 & \\
Two $\varepsilon 4$ & 0 & 12 & 6 & \\
\end{tabular}

Values are showed as mean $( \pm \mathrm{SD})$, Mini Mental State Examination(MMSE), fluorodeoxyglucose (FDG)-positron emission tomography $(P E T)$, results of ANOVA analysis between groups noted as $\mathrm{p}$ value

Table2. Significant Results of partial Correlation Analyses of DTI metrics and CSF P tau 181 levels among all participants

\begin{tabular}{|c|c|c|c|c|}
\hline Regions & $\begin{array}{l}\text { Fractional } \\
\text { anisotropy }\end{array}$ & Mean diffusivity & Radial diffusivity & Axial diffusivity \\
\hline & Correlation & Correlation & Correlation & Correlation \\
\hline & Coefficient & Coefficient & Coefficient & Coefficient \\
\hline Left hippocampal cingulum & $-0.218 * *$ & 0.164 & $0.182^{*}$ & 0.124 \\
\hline Splenium of right corpus callosum & $-0.142 *$ & $0.171 *$ & $0.168 *$ & $0.163 *$ \\
\hline Splenium of left corpus callosum & -0.101 & $0.141 *$ & 0.133 & $0.152 *$ \\
\hline Left Tapatum & $-0.167 *$ & $0.166^{*}$ & $0.169 *$ & $0.156 *$ \\
\hline Right Tapatum & $-0.175^{*}$ & $0.189 * *$ & $0.194^{* *}$ & $0.174 *$ \\
\hline Left Medial lemniscus & 0.048 & $-0.178 *$ & $-0.163 *$ & $-0.174 *$ \\
\hline Left Posterior corona radiata & 0.069 & 0.123 & 0.102 & 0.139 \\
\hline Right Posterior corona radiata & 0.074 & $0.188^{* *}$ & $0.147 *$ & $0.223 * *$ \\
\hline Right Sagittal stratum & -0.002 & $0.181 *$ & $0.164 *$ & $0.199 * *$ \\
\hline Right Uncinate fasciculus & -0.134 & $0.199 *$ & $0.202 * *$ & $0.188^{* *}$ \\
\hline $\begin{array}{l}\text { Retrolenticular part of right internal } \\
\text { capsule }\end{array}$ & -0.030 & $0.192 * *$ & $0.178 *$ & $0.189 * *$ \\
\hline
\end{tabular}


$* \mathrm{p}<0.05$

$* * p<0.01$

$* * * \mathrm{p}<0.001$

Partial correlation coefficient of DTI metrics value of the brain regions and plasma p tau 181 levels controlled for age, APOE, and sex.

TABLE3. Significant Results of partial Correlation Analyses of DTI metrics and Plasma p tau 181 levels within groups

\begin{tabular}{|c|c|c|c|c|}
\hline Regions & $\begin{array}{l}\text { Fractional } \\
\text { anisotropy }\end{array}$ & Mean diffusivity & Radial diffusivity & Axial diffusivity \\
\hline & $\begin{array}{l}\text { Correlation } \\
\text { Coefficient }\end{array}$ & $\begin{array}{l}\text { Correlation } \\
\text { Coefficient }\end{array}$ & $\begin{array}{l}\text { Correlation } \\
\text { Coefficient }\end{array}$ & $\begin{array}{l}\text { Correlation } \\
\text { Coefficient }\end{array}$ \\
\hline Alzheimer's patients & & & & \\
\hline Left Inferior cerebellar peduncle & 0.233 & $-0.341 *$ & -0.341 & $-0.335^{*}$ \\
\hline Left Medial lemniscus & -0.107 & $-0.420 * *$ & $-0.351 *$ & $-0.469 * *$ \\
\hline $\begin{array}{l}\text { Right Superior cerebellar } \\
\text { peduncle } \\
\text { Mild Cognitive Impairments }\end{array}$ & 0.258 & $-0.382^{*}$ & $-0.374 *$ & -0.379 \\
\hline $\begin{array}{l}\text { Left Medial lemniscus } \\
\text { Healthy Controls }\end{array}$ & 0.138 & $-0.138 *$ & $-0.185^{*}$ & -0.144 \\
\hline $\begin{array}{l}\text { Right Superior cerebellar } \\
\text { peduncle }\end{array}$ & 0.171 & 0.227 & 0.140 & $0.343^{*}$ \\
\hline Left Uncinate fasciculus & -0.289 & 0.319 & 0.333* & 0.279 \\
\hline
\end{tabular}

$* \mathrm{p}<0.05$

$* * \mathrm{p}<0.001$

partial correlation coefficient of DTI metrics value of the brain regions and plasma p tau 181 levels controlled for age, APOE, and sex. 